\title{
Central coordination as an alternative for local coordination in a multicenter randomized controlled trial: the FAITH trial experience
}

Stephanie M Zielinski ${ }^{1}$, Helena Viveiros ${ }^{2}$, Martin J Heetveld ${ }^{3}$, Marc F Swiontkowski ${ }^{4}$, Mohit Bhandari ${ }^{2}$, Peter Patka ${ }^{1,5}$ and Esther MM Van Lieshout ${ }^{*}$, for on behalf of the FAITH trial investigators

\begin{abstract}
Background: Surgeons in the Netherlands, Canada and the US participate in the FAITH trial (Fixation using Alternative Implants for the Treatment of Hip fractures). Dutch sites are managed and visited by a financed central trial coordinator, whereas most Canadian and US sites have local study coordinators and receive per patient payment. This study was aimed to assess how these different trial management strategies affected trial performance.
\end{abstract}

Methods: Details related to obtaining ethics approval, time to trial start-up, inclusion, and percentage completed follow-ups were collected for each trial site and compared. Pre-trial screening data were compared with actual inclusion rates.

Results: Median trial start-up ranged from 41 days (P25-P75 10-139) in the Netherlands to 232 days (P25-P75 98423) in Canada ( $p=0.027$ ). The inclusion rate was highest in the Netherlands; median 1.03 patients (P25-P75 0.432.21) per site per month, representing $34.4 \%$ of the total eligible population. It was lowest in Canada; 0.14 inclusions (P25-P75 0.00-0.28), representing 3.9\% of eligible patients $(p<0.001)$. The percentage completed followups was $83 \%$ for Canadian and Dutch sites and 70\% for US sites ( $p=0.217$ ).

Conclusions: In this trial, a central financed trial coordinator to manage all trial related tasks in participating sites resulted in better trial progression and a similar follow-up. It is therefore a suitable alternative for appointing these tasks to local research assistants. The central coordinator approach can enable smaller regional hospitals to participate in multicenter randomized controlled trials. Circumstances such as available budget, sample size, and geographical area should however be taken into account when choosing a management strategy.

Trial Registration: ClinicalTrials.gov: NCT00761813

Keywords: randomized controlled trial, management, trial coordinator, trial performance, inclusion, follow-up

\section{Background}

Randomized controlled trials (RCTs) are generally perceived as the reference standard for generating valid scientific evidence on the evaluation of medical treatments and interventions [1]. Unfortunately, RCTs continue to be relatively scarce in the orthopedic trauma literature [2]. This may be attributed to the logistical challenges of the implementation of RCTs.

\footnotetext{
* Correspondence: e.vanlieshout@erasmusmc.nl

'Dept. of Surgery-Traumatology, Erasmus MC, University Medical Center

Rotterdam, P.O. Box 2040, 3000 CA Rotterdam, the Netherlands

Full list of author information is available at the end of the article
}

One of the most apparent challenges of RCTs is to recruit the required number of patients as timely and efficiently as possible [3]. Availability of fewer patients than expected usually leads to an extended trial period and increased costs. Multicenter collaborations offer the potential of recruiting more patients within a shorter time period, which can be advantageous if large patient numbers are required or if the targeted population has a low incidence. They also have the advantage of increased generalizability of the results $[4,5]$. However, the conduct of multicenter clinical trials requires a

\section{Biomed Central}


complex organization, which applies even more to international trials $[6,7]$.

Obtaining ethics or Institutional Review Board (IRB) approval is a potential cause of delay, as procedures, documents, and legislation may vary between countries [8]. This process is often time-consuming and it is recommended to have dedicated and well-trained study personnel available to assist participating sites with these administrative tasks [9-13].

Another challenge in multicenter research is the selection and recruitment of appropriate participating clinical sites. Every participating site should have a devoted and dedicated clinician as site principal investigator. As surgeons often lack the time to spend on research, it is important to have an assisting research team that can adopt many of the time consuming research tasks. Having adequate support can be more important for sites to decide to participate, than offering a financial compensation for participation $[14,15]$. The presence of a trial coordinator or assistant will also facilitate an appropriate study infrastructure, which is a requirement for proper study conduct $[3,8,16-18]$. As community hospitals generally lack such infrastructure, they often cannot participate in multicenter trials. This is unfortunate, as some injuries or interventions are much more frequent in community hospitals than in university hospitals. Their participation in a multicenter trial could therefore positively influence the recruitment rate. Once a large multicenter or multinational RCT has started, a relatively complex organization should be implemented and maintained in order to assure a complete patient follow-up and a high quality in data management $[19,20]$.

Usually individual sites are required to manage their local ethics procedures and trial logistics. They generally receive per patient payments as compensation. As an alternative option, a single trial coordinator can be appointed to manage all trial-related tasks for multiple sites in a certain geographic area or country, in which travel time is limited and does not include air fares. This is dependent on availability of full financial support, but can relieve local sites from many trial related tasks. This may attract more sites to participate, provide a smoother process of obtaining ethics approval, quicker trial start-up, higher inclusion rates and follow-up completeness.

Similar management strategies have been applied in the FAITH trial (Fixation using Alternative Implants for the Treatment of Hip fractures, NCT00761813). The FAITH trial is an international multicenter study initiated by the IHFRC (International Hip Fracture Research Collaborative) [21]. The primary objective of this trial is to assess the impact of sliding hip screws versus cancellous screw fixation on rates of revision surgery at two years in elderly patients with femoral neck fractures. This trial has been launched in over 60 sites, predominantly situated in the Netherlands, Canada, and the US. All sites in the Netherlands are managed and visited by a single financed national trial coordinator. Most Canadian and US sites have individual local site coordinators and receive per patient payments. The aim of this study was to assess how these strategies affected performance of the FAITH trial.

\section{Methods}

\section{Study Characteristics}

In the Netherlands 14 hospitals participated in the FAITH trial. In Canada 11 hospitals participated, and in the US 29. Besides patient enrolment, sites were also required to register all patients that were excluded or missed for inclusion.

In the period before the trial started 26 of the participating Dutch, Canadian and US sites started prospective screening for patients during a short defined period to explore the amount and rate of potential inclusions that could be expected from each site. The results of this prospective screening study were used for the further planning of the definitive trial.

Two different strategies were applied for the organization and management of the FAITH trial in these participating countries. In the Netherlands the trial was coordinated and managed from a university hospital. One central, national trial coordinator was appointed upon obtaining adequate funding. This was a medical doctor working on her $\mathrm{PhD}$ project. This coordinator was responsible for all study related tasks at all fourteen participating sites. She arranged the process of ethics approval and the necessary documents for all sites, initiated study start-up, maintained communication with sites and the methods center, randomized all patients, and collected all follow-up data for 250 patients. During a period when study related tasks became too much to handle for a single person, she was assisted by the research team at the central coordinating site. There was no local research support at the participating sites. The coordinator travelled regularly to all participating sites, which were within a range of maximum 114 kilometers from the coordinating site. Participating sites were only responsible for patient selection. Sites received no payment; all funding was used for covering costs of the central trial coordination.

Most Canadian and US sites (located in various states ranging from Nova Scotia to British Columbia and from California to New York) were responsible themselves for all local study related tasks. The vast majority of these sites have individual, local, research assistants that take care of these tasks for the FAITH trial, as well as for other trials in that hospital. As compensation these sites 
receive per patient payment. In these countries there was no central, national trial coordinator.

All countries were supervised by the FAITH trial central methods center and steering committee. In order to monitor progress and to keep participating sites focused, there was contact between the methods center and the Dutch central coordinator, Canadian and US local principal investigators on a weekly base. The Dutch central coordinator had weekly contact with the participating sites and all sites received monthly newsletters showing the progress of the trial.

\section{Data}

Data related to trial initiation, organization, and performance were collected up to August 11, 2010. At this time patient recruitment was still ongoing in Canada and the US. Data were collected concerning:

- baseline characteristics; country, type of hospital (i.e., university, non-university teaching, or non-university nonteaching), type of research coordinator (i.e., not available, site-specific or provided for by central coordinating site);

- process of obtaining ethics approval; submission and approval date, number of submissions and type of revisions (i.e., changes in wording or content of the informed consent form, changes in in- or exclusion criteria, changes in the wording or content of the study protocol, extra information on the study protocol and procedures, extra information on financial aspects, or request for additional documents);

- pre-trial screening period; screening start and stop date, total number of patients screened and number of eligible patients screened;

- trial period: trial start and (if applicable) stop dates, total number of included, excluded and missed patients that were registered, total number of patients that were missed for registration (for the Netherlands only), total amount of kilometers travelled by research coordinator and associated costs (for the Netherlands only), followup completeness.

Additional variables that were calculated from these data are described in Table 1.

\section{Data Analysis}

All analyses were conducted using SPSS (version 16.0, SPSS Inc., Chicago, IL, USA). Data from the three countries (the Netherlands, Canada and US) were compared. The choice to compare these three countries was made because of the differences in trial management between the Netherlands and Canada/US described above. Comparing these countries separately also allowed the possibility to study country related differences that may affect trial performance, independent from the trial management strategy chosen. Continuous variables are presented as median with interquartile ranges. Categorical variables are presented as number (percentage). Continuous variables were compared with the Kruskal-Wallis Analysis of Variance (ANOVA). Post-hoc pair wise comparisons were performed using the Mann-Whitney U-test. Categorical variables were compared with the Chi-squared test. A P-value $<0.05$ (twosided) was taken as threshold of statistical significance.

\section{Results}

Characteristics of participating sites

The type of hospitals participating was similar for the Netherlands (NL), Canada (CA) and the US (Table 2). All sites in the Netherlands were centrally coordinated, whereas local site coordination was available at $72.7 \%$ of Canadian sites and $96.6 \%$ of US sites.

Table 1 Additional variables calculated concerning the period of obtaining ethics approval and the screening and trial period

\begin{tabular}{ll}
\hline Variable & Calculation \\
\hline Time necessary for ethics/IRB approval (days) & $a-b$ \\
Time between ethics/IRB approval and start trial (days) & $c-a$ \\
Screening period (days) & $f-e$ \\
Enrolment/trial period (days) & $d-c$ \\
Total number of patients in screening period (n per month) & $g /(f-e)$ \\
Number of eligible patients in screening period (n per month) & $\mathrm{h} /(\mathrm{f}-\mathrm{e})$ \\
Proportion eligible patients of total in screening period (\%) & $(\mathrm{h} / \mathrm{g})^{*} 100$ \\
Total number of patients per month in trial period ( $\mathrm{n}$ per month) & $(\mathrm{i}+\mathrm{j}) /(\mathrm{d}-\mathrm{c})$ \\
Number of inclusions in trial period (n per month) & $\mathrm{i} /(\mathrm{d}-\mathrm{c})$ \\
Proportion inclusions of total in trial period (\%) & $(\mathrm{i} /(\mathrm{i}+\mathrm{j})) * 100$ \\
Proportion patients that were missed for registration in trial period of total $(\%)$ & $(\mathrm{k} /(\mathrm{i}+\mathrm{j}+\mathrm{k})) * 100$ \\
Rate of total number of patients per month in trial period versus screening period & $((\mathrm{i}+\mathrm{j}) /(\mathrm{d}-\mathrm{c})) /(\mathrm{g} /(\mathrm{f}-\mathrm{e}))$ \\
Rate of number of inclusions/eligible patients per month in trial period versus screening period & $(\mathrm{i} /(\mathrm{d}-\mathrm{c})) /(\mathrm{h} /(\mathrm{f}-\mathrm{e}))$ \\
Rate of percentage inclusions/eligible patients per month in trial period versus screening period & $((\mathrm{i} /(\mathrm{i}+\mathrm{j}) * 100) /((\mathrm{h} / \mathrm{g}) * 100)$
\end{tabular}

a, ethics/IRB approval date; b, ethics/IRB submission date; $c$, trial start date; $d$, trial stop date; e, screening start date; $f$, screening stop date; $g$, total number of patients screened; $h$, number of eligible patients screened; $i$, number of inclusions; $j$, number of excluded or missed patients that were registered; $k$, number of patients that were missed for registration. 
Table 2 Characteristics of countries participating in the FAITH trial

\begin{tabular}{lllll}
\hline & $\begin{array}{l}\mathbf{N L} \\
\mathbf{( N = 1 4 )}\end{array}$ & $\begin{array}{l}\mathbf{C A} \\
\mathbf{( N = 1 1 )}\end{array}$ & $\begin{array}{l}\text { US } \\
\mathbf{( N = 2 9 )}\end{array}$ & P-value \\
\hline $\begin{array}{l}\text { Type of hospital } \\
\quad \text { University }\end{array}$ & $4(28.6)$ & $9(81.8)$ & $16(55.2)$ & 0.051 \\
$\quad$ Non-university teaching & $10(71.4)$ & $2(18.2)$ & $11(37.9)$ & $2(6.9)$ \\
$\quad 0(0.0)$ & $0(0.0)$ & & $1(3.4)$ \\
$\quad$ Non-university non-teaching & $0(0.0)$ & $0(0.0)$ & $28(96.6)$ & 0.001 \\
Trial coordinator & $0(0.0)$ & $8(72.7)$ & $0(0.0)$ & \\
$\quad$ Not available & $14(100.0)$ & $3(27.3)$ & & \\
$\quad$ Available at site & & & \\
\hline
\end{tabular}

NL, the Netherlands; CA, Canada; US, United States.

Numbers in the headers represent the number of sites participating per country.

Data are presented as numbers with percentage between brackets. Statistics were calculated using the Chi-squared test.

\section{Process of obtaining ethics/IRB approval}

The time necessary for ethics/IRB approval was significantly longer in the Netherlands (median 104 days) than in Canada (median 55 days) and the US (median 53 days; $\mathrm{p}=0.027$; Table 3 ). For all countries the median number of submissions requested by the ethics committee was one. However, due to the differences in data distribution and skewness there was still a significant difference in requested submissions between NL (P25P75 0.0-1.0) and the US (P25-P75 1.0-3.0) ( $\mathrm{p}=0.014)$. The type of revisions requested did not differ between the countries; the vast majority concerned wording and content of the informed consent form.

\section{Pre-trial screening period}

Of the currently participating FAITH sites four Dutch, eight Canadian, and fourteen US sites also took part in the pre-trial prospective screening period. The duration of the screening period did not differ significantly between groups (Table 4). The number of patients screened per site was least in the Netherlands with six patients in total and 3.3 patients per month. Forecasted total number of patients was highest for Canadian sites with 15 patients per site in total and 7.5 per month $(\mathrm{p}=$ 0.006 and $p=0.016$ ). Other variables, concerning the amount and proportion of eligible patients screened, were not significantly different between countries.

\section{Trial period}

Dutch hospitals started enrolment in the period between February 2008 and October 2008. In August 2009 the national goal of enrolling 250 patients was achieved. Canadian hospitals started enrolment between March 2008 and June 2010. In the US hospitals started

Table 3 Data concerning the process of obtaining ethics/IRB approval of countries participating in the FAITH trial

\begin{tabular}{|c|c|c|c|c|}
\hline & $\begin{array}{l}\mathrm{NL} \\
(\mathrm{N}=14)\end{array}$ & $\begin{array}{l}\text { CA } \\
(N=11)\end{array}$ & $\begin{array}{l}\text { US } \\
(N=19)\end{array}$ & P-value \\
\hline Time necessary for ethics/IRB approval' (days) & $\begin{array}{l}104 \\
(74-135)\end{array}$ & $\begin{array}{l}55 \\
(27-77)\end{array}$ & $\begin{array}{l}53 \\
(44-105)\end{array}$ & $0.027^{+a}$ \\
\hline Revision rounds ${ }^{1}$ & $1(0.0-1.0)$ & $1(0.8-1.0)$ & $1(1.0-3.0)$ & $0.014^{+b}$ \\
\hline \multicolumn{5}{|l|}{ Type of revisions requested: } \\
\hline Wording of IC Form ${ }^{2}$ & $6(42.9)$ & $5(50.0)$ & $12(66.7)$ & $0.382^{++}$ \\
\hline Content of IC Form ${ }^{2}$ & $6(42.9)$ & $5(50.0)$ & $8(44.4)$ & $0.938^{++}$ \\
\hline In- or exclusion criteria ${ }^{2}$ & $0(0.0)$ & $1(10.0)$ & $1(5.3)$ & $0.511^{++}$ \\
\hline Wording of study protocol ${ }^{2}$ & $0(0.0)$ & $1(10.0)$ & $0(0.0)$ & $0.185^{++}$ \\
\hline Content of study protocol ${ }^{2}$ & $1(7.1)$ & $2(20.0)$ & $1(5.3)$ & $0.406^{++}$ \\
\hline Additional information in study protocol/procedures ${ }^{2}$ & $5(35.7)$ & $3(30.0)$ & $7(36.8)$ & $0.932^{++}$ \\
\hline $\begin{array}{l}\text { Financial aspects - } \\
\text { Request for extra information }{ }^{2}\end{array}$ & $0(0.0)$ & $1(10.0)$ & $3(15.8)$ & $0.303^{++}$ \\
\hline Request additional documents ${ }^{2}$ & $2(14.3)$ & $0(0.0)$ & $3(15.8)$ & $0.421^{++}$ \\
\hline
\end{tabular}

NL, the Netherlands; CA, Canada; US, United States.

Numbers in the headers represent the number of sites per country for which data were available.

IC, informed consent form.

${ }^{1}$ Data are presented as median with $\mathrm{P}_{25}-\mathrm{P}_{75}$ given between brackets. ${ }^{2}$ Data are presented as number with percentages.

${ }^{+}$Kruskal-Wallis ANOVA, ${ }^{++}$Chi-squared test

Post-hoc pair wise comparisons were performed using the Mann-Whitney U-test: ${ }^{a}$ Statistical significance was reached when comparing NL vs. CA ( $p=0.025$ ), and NL vs. US ( $p=0.019), C A$ vs. US: not significant. ${ }^{b}$ Statistical significance was reached when comparing NL vs. US ( $\left.p=0.007\right)$, other groups: not significant 
Table 4 Data concerning the pre-trial screening period of countries participating in the FAITH trial

\begin{tabular}{lllll}
\hline & $\begin{array}{l}\mathbf{N L} \\
(\mathbf{N}=\mathbf{4})\end{array}$ & $\begin{array}{l}\mathbf{C A} \\
\mathbf{( N = 8 )}\end{array}$ & $\begin{array}{l}\text { US } \\
\mathbf{( N = 1 4 )}\end{array}$ & P-value \\
\hline Screening period (days) & $55(51-92)$ & $60(56-74)$ & $50(20-69)$ & 0.121 \\
Eligible patients (N) & $4(3-6)$ & $4(1-10)$ & $3(1-6)$ & 0.571 \\
Total patients (N) & $6(6-9)$ & $15(15-34)$ & $6(5-11)$ & $0.006^{\mathrm{a}}$ \\
Eligible patients (N per month) & $2.0(1.7-2.4)$ & $1.5(0.7-4.1)$ & $2.2(1.1-5.0)$ & 0.786 \\
Total patients (N per month) & $3.3(3.0-3.6)$ & $7.5(5.6-17.4)$ & $5.0(3.1-8.2)$ & $0.016^{b}$ \\
Proportion eligible patients (\% of total) & $63(53-67)$ & $14(10-40)$ & $48(29-71)$ & 0.062 \\
\hline
\end{tabular}

$\mathrm{NL}$, the Netherlands; CA, Canada; US, United States.

Numbers in the headers represent the number of sites per country that data were available for.

Data are presented as median with $\mathrm{P}_{25}-\mathrm{P}_{75}$ given between brackets.

Statistics were calculated using the Kruskal-Wallis ANOVA.

Post-hoc pair wise comparisons were performed using Mann-Whitney U-test: ${ }^{\text {a }}$ Statistical significance was reached when comparing NL vs. CA ( $\left.p=0.009\right)$, and CA vs. USA $(p=0.004)$, NL vs. USA: not significant. ${ }^{b}$ Statistical significance was reached when comparing NL vs. CA ( $\left.p=0.007\right)$, other groups: not significant.

enrolment between February 2009 and September 2010. In Canada and the US enrolment is still ongoing.

The time necessary for trial start-up was defined as the time between obtained ethics approval and the actual trial start up. With a median of 41 days, trial start-up was fastest for the Netherlands. Trial start-up took more than five times longer for Canada (median 232 days; $\mathrm{p}=0.027$; Table 5). Because the median enrolment period was statistically significantly longer for the US than for the Netherlands (median 283 vs. 423 days, respectively, $\mathrm{p}=0.001$ ), crude numbers were also calculated per month. The total number of patients seen per month in the trial period was similar in all countries; however, the inclusion rate in the Dutch sites per month was more than three times higher than in the US sites, and more than seven times higher compared with Canadian sites (1.03 patients per month vs. 0.31 and 0.14 , respectively, $\mathrm{p}<0.001)$. Inclusion progression of all countries is also shown in Figure 1. Similar differences were seen when comparing the proportion inclusions of the total patient group. In Dutch sites $34.4 \%$ of the patients were included vs. $16.7 \%$ in US sites and $3.93 \%$ in Canadian sites $(\mathrm{p}=0.001)$. These numbers may however be influenced by the varying screening compliance in all countries. For example, sites screening all hip fractures would certainly have greater screening failure rates then those sites screening only those hip fractures that were deemed treatable by internal fixation.

For sites in the Netherlands data were extracted from hospital records in order to check for omissions in

Table 5 Data concerning the trial period of countries participating in the FAITH trial

\begin{tabular}{|c|c|c|c|c|}
\hline & $\begin{array}{l}\mathrm{NL} \\
(\mathrm{N}=14)\end{array}$ & $\begin{array}{l}C A \\
(N=11)\end{array}$ & $\begin{array}{l}\text { US } \\
(N=29)\end{array}$ & P-value \\
\hline Time between ethics approval and start trial (days) & $41(10-139)$ & $232(98-423)$ & $87(45-255)$ & $0.027^{a}$ \\
\hline Enrolment period (days) & $423(381-509)$ & $482(267-663)$ & $283(142-360)$ & $0.001^{b}$ \\
\hline Inclusions & $13(7-27)$ & $3(0-5)$ & $3(1-6)$ & $<0.001^{c}$ \\
\hline Registered patients & $23(12-36)$ & $54(3-75)$ & $16(5-27)$ & 0.060 \\
\hline Patients missed for registration ${ }^{1}$ & $35(10-81)$ & Unknown & Unknown & \\
\hline Inclusions (n per month) & $1.03(0.43-2.21)$ & $0.14(0.00-0.28)$ & $0.31(0.09-0.62)$ & $<0.001^{\mathrm{d}}$ \\
\hline Total patients (n per month) & $2.49(1.60-3.64)$ & $2.76(0.60-8.75)$ & $1.96(1.11-3.75)$ & 0.574 \\
\hline Proportion inclusions (\% of total) & $34.4(23.8-62.6)$ & $3.93(0.00-13.2)$ & $16.7(2.50-31.3)$ & $0.001^{e}$ \\
\hline Proportion patients that were missed for registration of total ${ }^{1}(\%)$ & $57.4(32.2-65.2)$ & Unknown & Unknown & \\
\hline Completed follow-ups (\%) & $82.6(80.0-84.6)$ & $83.5(72.7-95.2)$ & $70.0(60.0-88.1)$ & 0.217 \\
\hline Follow-ups in window (\%) & $77.1(71.0-82.2)$ & $85.9(81.0-95.0)$ & $85.7(70.0-100.0)$ & 0.073 \\
\hline
\end{tabular}

NL, the Netherlands; CA, Canada; US, United States.

Numbers in the headers represent the number of sites per country that data were available for.

Data are presented as median with $\mathrm{P}_{25}-\mathrm{P}_{75}$ given between brackets.

Statistics were calculated using the Kruskal-Wallis ANOVA.

1 Data available for NL only.

Post-hoc pair wise comparisons were performed using a Mann-Whitney U-test: ${ }^{\text {a }}$ Statistical significance was reached when comparing NL vs. CA ( $p=0.010$ ), other groups: not significant. ${ }^{b}$ Statistical significance was reached when comparing NL vs. US $(p<0.001)$, other groups: not significant. ${ }^{c}$ Statistical significance was reached when comparing NL vs. CA $(p=0.002)$ and NL vs. US $(p<0.001)$, CA vs. US: not significant. ${ }^{d}$ Statistical significance was reached when comparing NL vs. CA $(p=0.001)$ and NL vs. US ( $p=0.001), C A$ vs. US: not significant. ${ }^{e}$ Statistical significance was reached when comparing NL vs. CA ( $\left.p<0.001\right)$ and NL vs. US ( $p$ $=0.009$ ), CA vs. US: not significant. 


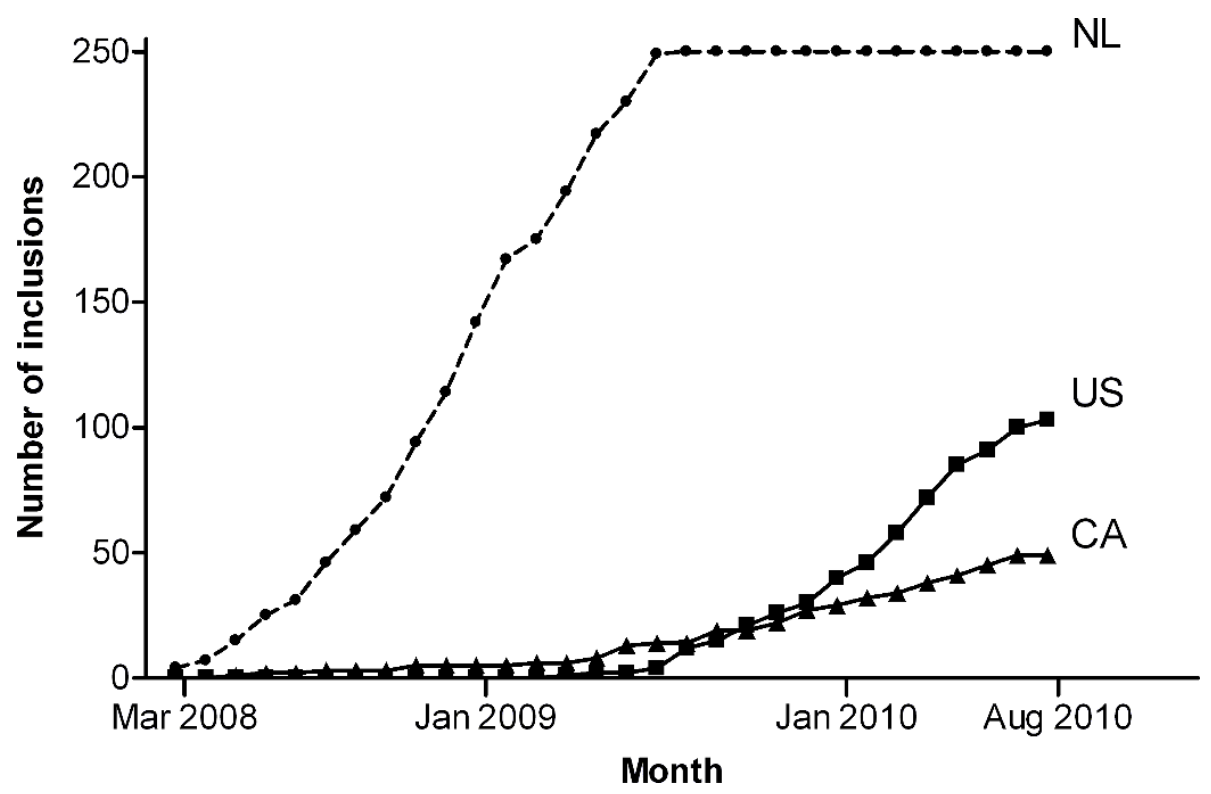

Figure 1 Inclusion progression for the Netherlands (NL), Canada (CA) and the United States (US).

patient registrations during the trial. A median of 35 excluded and missed patients (P25-P75 10-81) were not registered per site, despite clear instructions to participating sites that this was required for the trial. This represented $57.4 \%$ of the total amount of patients seen during the trial period. These data were not available for the other countries.

Follow-up data were collected at eight time points, four times in clinic and four times by telephone. The percentage completed follow-ups were calculated for all time points and the overall percentage completed follow-ups was computed. The median overall percentage completed follow-ups was $70 \%$ in Canada, and exceeded $80 \%$ in the Netherlands and US. No statistically significant differences were found between countries (Table 5). The median percentage of follow-ups that were completed within the predefined acceptable time window was $77 \%$ in the Netherlands, and $86 \%$ in Canada and the US. Again, this was not statistically significantly different (Table 5).

During this study, the central trial coordinator in the Netherlands travelled 28,842 kilometers in order to visit all fourteen participating sites for trial start-up, enrolment, and data collection in clinic. This resulted in $€$ 9,771 total travel costs.

\section{Pre-trial screening period versus trial period}

Pre-trial screening data regarding eligible patients were compared with the actual inclusion rates and percentages in the trial period, to study the value of pre-trial screening data. An overview of the calculated variables is shown in Table 1 . Inclusion rates in the trial were much lower than expected from the screening period: a decline of $67 \%$ (P25-P75 42-83) was noted for sites in the Netherlands versus a decline of 92\% (P25-P75 78100) for US and 93\% (P25-P75 68-100) for Canadian sites $(\mathrm{p}=0.154$; Figure 2$)$. The total number of patients seen in the trial period versus the screening period also displayed a decline: $41 \%$ less (P25-P75 -2-62) for the Netherlands versus 52\% (P25-P75 9-88) for Canada and 69\% (P25-P75 35-81) for the US ( $\mathrm{p}=0.477$ ). Consequently, the proportion inclusions of the total patient group also decreased: 48\% decrease (P25-P75 14-69) for sites in the Netherlands versus 83\% (P25-P75 39-100) for Canadian sites and 83\% (P25-P75 56-100) for US sites $(\mathrm{p}=0.091)$.

\section{Discussion}

In this study, trial progression in the Netherlands, where a central trial coordinator managed most tasks for multiple centers in a restricted geographical area, was better than in Canada and the US, where local research assistants were appointed at individual sites. The central trial coordinator system was associated with a shorter trial start-up time, higher inclusion rate, and a higher inclusion percentage. Collection of follow-up data was equally good in both systems.

The process of obtaining ethics approval can be timeconsuming and stressful and may yield diverse responses from ethics committees [9-13]. For the FAITH trial, obtaining ethics approval took longer in the Netherlands than in Canada or the US. This may have been influenced by the time that the trial coordinator or sites needed to assemble forms for the ethics committee. 


\section{Total}

Figure 2 The percentage decline in total number of patients seen per month, number of inclusions per month and percentage inclusions of total number of patients, during the trial period in comparison with the pre-trial screening period. To calculate this percentage decline, the total number of patients seen in the trial period was divided by the total number of patients seen in the pre-screening trial period. Similar calculations were made for the number of inclusions per month and the inclusion percentage. These rates (a) were transformed to a percentage decline (b) using the following formula: $b=(1-a) * 100 \%$. This figure therefore shows that for all variables there were fewer patients during the trial period compared with the pre-trial screening period in all countries. NL, the Netherlands; CA, Canada; US, United States.

Ethics submissions may have taken longer in the Netherlands at the very start of the trial, when there was no central trial coordinator yet. The longer approval process in the Netherlands may also have been caused by more inefficient medical ethics procedures [10]. In the Netherlands multicenter studies need approval from a central ethics committee that performs a full review of the study documents. Subsequently, ethics committees in participating sites should only advise on local feasibility. This two-step approach was aimed to simplify and shorten the ethics procedures for multicenter trials. It is nevertheless disputable if this goal is currently achieved [10]. In Canada and the US a single procedure for study review and approval is performed at all sites, which may turn out to be more efficient.

The number of resubmissions requested by the ethics committees was lower in the Netherlands, and can therefore not have contributed to the prolonged approval process. The availability of a central trial coordinator with detailed insight and knowledge on the study content could have prevented incorrect submissions in the Netherlands. In all countries remarks of the ethics committees mainly involved the informed consent form and extra information on the study protocol and procedures. Standardization of the ethics approval process is recommended as it may reduce the local differences in ethics approval terms.

The trial start-up period was statistically significantly shorter in the Netherlands (median 41 days) than in the US and Canada (87 and 232 days). In these latter two countries contract negotiations with participating sites had to take place during the trial start-up period, whereas this procedure was not applicable in the Netherlands. Furthermore, research assistants from Canada and the US frequently reported a long period between grant approval and official release of the funds. These aspects related to the per patient payment strategy applied in Canada and the US slowed the trial start-up process in these countries. In the Netherlands contract negotiations or per patient payments were unnecessary, as all tasks were performed by the central study coordinator, not resulting in delay. The assistance of the Dutch central trial coordinator in trial start-up activities (e.g. distributing study materials, giving start-up presentations) may also have contributed to a more efficient and speedy trial start-up.

Most influential to the differences in trial progression between the countries were the evident differences in inclusion rate and percentage, which were statistically significantly higher for sites in the Netherlands. The availability of the central trial coordinator in the Netherlands made it possible for smaller, non-university sites without a local research infrastructure or coordinator to participate. These sites generally treat more patients from the targeted population (i.e., femoral neck fracture patients) than the large university hospitals, but would normally not have been able or willing to participate in the trial, because they lack a local coordinator. Most of the principal investigators of these sites reported to be very motivated to enroll patients, as the (administrative) 
burden of participation was relatively low, thanks to the fact that the performance of follow-ups and other trial related tasks were adopted by the central trial coordinator. They declared that this was crucial in their decision to participate. This high devotion and lack of burdensome trial related tasks will probably have contributed to a good inclusion rate. Therefore, the availability of a central trial coordinator can contribute to a fast enrolment, both directly, by motivating local principal investigators to participate and enroll because of the low (administrative) burden of participation, and indirectly, by enabling high-volume hospitals without a local research network to participate. However, differences in accrual between countries may also have been affected by the known intercultural differences in preferred treatments for femoral neck fractures [22]. North American surgeons may have considered less femoral neck fracture patients suitable for internal fixation than Dutch surgeons do, as they are less committed to internal fixation as a preferred treatment. Moreover, surgeons from the US and Canada reported at investigator meetings that North American patients may be less lenient to participate in trials and that they experienced problems at obtaining informed consent [23]. Within the countries, higher accrual was also associated with a large target population, dedicated and compliant principal investigators, and low study related workload for participating surgeons. These are important aspects to pursue when planning a multicenter randomized controlled trial, and can be facilitated by appointing a central trial coordinator.

Comparison of the pre-trial prospective screening data with the actual trial data showed an obvious discrepancy in all countries. It is known that participating surgeons tend to overestimate enrolment numbers based upon a pre-trial screening period [24]. In this study accrual was also much lower than expected from the screening period. The use of pre-trial screening can therefore be debated. However, it may be useful to indicate good dedication to participate and help raise awareness for the upcoming study in potential sites. If a pre-trial screening is deemed necessary, a retrospective approach is recommended, as it is easier and results in similar estimated numbers, compared with a prospective approach [24].

The percentage completed follow-ups was not affected by the availability of a central trial coordinator and was between $70 \%$ and $84 \%$ for all countries. Follow-ups did seem to be completed within the window a bit less in the Netherlands, although not statistically significant. This was a result of the limitations that we experienced from the central trial coordinator approach. Usefulness of this approach will decrease with an increased sample size and an increased distance between sites. A single person can only manage a certain maximum number of sites and patients. Similarly, there is a maximum to the distance that can be traveled per day. In our study, a single study coordinator to manage 14 sites and 250 patients seemed somewhat limited. The study coordinator had to complete eight follow-ups per patient (four clinical and four telephonic), in 14 sites that were maximum $140 \mathrm{~km}$. apart (maximum 2 hours travel time), resulting in an average of almost $1,000 \mathrm{~km}$ traveled per week. In these circumstances it was not always feasible to manage all follow-ups. We feel that it would have been optimal to have one coordinator following a maximum number of 200 patients, in our study. It is also important to have a supporting research team available for assistance if work pressure gets too high for a single person. The central coordinator approach is feasible within European countries, as well as within American/ Canadian states.

Finally, a central coordinator may also contribute to the impartiality of the collected data and may prevent biases that could be introduced if the local coordinators/participating doctors are also the treating physician. Obviously, this could not be analyzed or proven in this study.

Obviously, this study has its limitations. Many of the results of this study were multifactorially influenced. Not all differences between countries can therefore be attributed to the difference in trial management system. If the two models (i.e., central management and local management) would have been conducted equally in each of the countries, bias due to country-specific conditions could have been ruled out. This was however impossible in the current study. Nevertheless, the availability of the central coordinator has certainly contributed to the speedier trial start-up, high enrolment rates and complete follow-up. Also, the limited number of sites available for data assemblage (especially for the screening period) and the fact that not all data were collected prospectively may have introduced some bias.

\section{Conclusions}

In summary, trial performance can be influenced by the management strategy chosen. This study shows that the appointment of a central financed trial coordinator to manage all trial related tasks is a feasible alternative for the more traditional approach of appointing trial related tasks to local research assistants at participating sites. Taking important circumstances such as available budget, sample size, and geographical area into account, a central trial coordinator approach can add to the success of a multicenter randomized trial. A central coordinator should be considered when studying injuries that occur more frequently in smaller regional hospitals (without a local research coordinator). It will enable 
these sites to participate in randomized controlled trials, resulting in a enhanced enrolment rate. It should also be considered when the targeted principal investigators are unable to participate due to the (administrative) burden of participation. However, a central coordinator should only be considered for multiple sites in a restricted geographical area. Depending upon the geographic spread of the sites and the frequency of followup a careful estimation should be made of the amount of patients and sites that can be managed by a single coordinator.

\section{Acknowledgements}

\section{Steering Committee}

Mohit Bhandari (chair), Philip J. Devereaux, Gordon Guyatt, Martin J. Heetveld, Kyle Jeray, Susan Liew, Martin J. Richardson, Emil H. Schemitsch, Marc Swiontkowski, Paul Tornetta III, Stephen Walter.

Methods Centre and Coordination of Canadian/International Sites Supported under the PSI and CIHR Grants

McMaster University, Hamilton, ON: Mohit Bhandari, Sheila Sprague, Helena Viveiros Nicole Simunovic, Diane Heels-Ansdell, Lisa Buckingham, Aravin Duraikannan.

Country Coordination of US Sites Supported under the NIH Grant University of Minnesota, Minneapolis, MN: Marc F. Swiontkowski, Julie Agel. Country Coordination of Dutch Sites Supported under the Fonds Nuts Ohra Grant

Erasmus MC, Rotterdam: Esther M.M. Van Lieshout, Stephanie M. Zielinski. Investigators - The Netherlands (site principal investigator is marked with an asterisk)

Academic Medical Center, Amsterdam: J Carel Goslings*, Robert Haverlag, Kees Jan Ponsen.

Erasmus MC, University Medical Center Rotterdam, Rotterdam: Peter Patka*, Martin G. Eversdijk, Rolf Peters, Dennis den Hartog, Oscar J.F. van Waes, Pim Oprel.

IJsselland Ziekenhuis, Capelle aan den IJssel: Piet A.R. de Rijcke*, C.L. Koppert, Steven E. Buijk, Richard P.R. Groenendijk, I. Dawson, G.W.M. Tetteroo, Milko M.M. Bruijninckx, Pascal G. Doornebosch, E.J.R. deGraaf Kennemer Gasthuis, Haarlem: Martin J. Heetveld*, Gijs A. Visser, Heyn Stockmann, Rob Silvis, J.P. Snellen, A. Rijbroek, Joris J.G. Scheepers, Erik G.J. Vermeulen, M.P.C. Siroen, Ronald Vuylsteke, H.L.F. Brom, H. Ryna. Medisch Centrum Haaglanden, Den Haag: Steven J. Rhemrev*, C.H.R. Bosman, Alexander de Mol van Otterloo, Jochem Hoogendoorn, Alexander C. de Vries, Sven A.G. Meylaerts.

Maasstad Ziekenhuis, Rotterdam: Gert R Roukema*, H. Josaputra, Paul Keller P.P. de Rooij, H. Kuiken, Han Boxma, B.I. Cleffken, Ronald Liem.

Onze Lieve Vrouwe Gasthuis, Amsterdam: Rudolf W. Poolman*, Maarten P. Simons, Frank H.W.M. van der Heijden, W.J. Willems, Frank R.A.J. de Meulemeester, Cor P. van der Hart, Kahn Turckan, Sebastiaan Festen, F. de Nies, Robert Haverlag, Nico J.M. Out, J. Bosma.

Reinier de Graaf Gasthuis, Delft: Maarten van der Elst*, Carmen C. van der Pol, Martijne van 't Riet, T.M. Karsten, M.R. de Vries, Laurents P.S. Stassen, Niels W.L. Schep, G.B. Schmidt, W.H. Hoffman.

St. Antonius Ziekenhuis, Nieuwegein: Michiel J.M. Segers*, Jacco A.C. Zijl, Bart Verhoeven, Anke B. Smits, J.P.P.M. de Vries, Bram Fioole, H. van der Hoeven, Evert B.M. Theunissen, Tammo S. de Vries Reilingh, Lonneke Govaert, Philippe Wittich, Maurits de Brauw, Jan Wille, Peter M.N.Y.M. Go, Ewan D. Ritchie, R.N. Wessel, Eric R. Hammacher.

St. Elisabeth Ziekenhuis, Tilburg: Michiel H.J. Verhofstad*, Joost Meijer, Teun van Egmond, Frank H.W.M. van der Heijden, Igor van der Brand. Tergooi Ziekenhuizen, Hilversum: Harm M van der Vis*, Martin Campo, Ronald Verhagen, G.H.R. Albers, A Zurcher.

University Medical Center St. Radboud, Nijmegen: A van Kampen, Jan Biert*, Arie B. van Vugt, Michael J.R. Edwards, Taco J. Blokhuis, Jan Paul M. Frölke, L. M.G. Geeraedts, J.W.M. Gardeniers, Edward T.C.H. Tan, L.M.S.J. Poelhekke, M.C. de Waal Malefijt, Bart Schreurs.
University Medical Center Utrecht, Utrecht: Rogier KJ. Simmermacher*, Jeroen van Mulken, Karlijn van Wessem, Taco J. Blokhuis, Steven M. van Gaalen, Luke P.H. Leenen.

Bronovo Ziekenhuis, Den Haag: Maarten W.G.A. Bronkhorst*, O.R. Guicherit. Investigators - Norway (site principal investigator is marked with an asterisk) Oslo University Hospital, Oslo: Frede Frihagen*, Lars Nordsletten, Ragnhild Oydna Stoen, Kine Brekke.

Investigators - Australia (site principal investigator is marked with an asterisk) Royal Brisbane and Women's Hospital, Herston, QLD: Kevin Tetsworth*, Patrick Weinrauch, Paul Pincus, Geoff Donald, Steven yang, Brett Halliday, Trevor Gervais, Michael Holt, Annette Flynn.

Royal Melbourne Hospital, Parkville, VIC: Marinis Pirpiris*, David Love, Andrew Bucknill, Richard J. Farrugia.

The Alfred, Melbourne, VIC: Susan Liew*, Adam Dowrick, Craig Donohue, Harvinder Bedi, Doug Li, Elton Edwards, Steven Csongvay, Russell Miller, Otis Wang, Andrew Chia, Russell Miller, Arvind Jain, Mathan Mammen, Zoe Murdoch, Claire Sage.

Investigators - India (site principal investigator is marked with an asterisk) University College of Medical Sciences - Guru Tegh Bahadur Hospital, Delhi: Anil Kumar Jain*, Amite Pankaj, Ajay Pal Singh.

Investigators - Colombia (site principal investigator is marked with an asterisk)

Fundación Sana Fe, Bogotá: Rodrigo Pesantez*, Adriana Martinez, Catherine Novoa.

Investigators - Canada (site principal investigator is marked with an asterisk) Foothills Medical Center, Calgary, AB: Richard E. Buckley*, Paul Duffy, Robert Korley, Kelly Johnston, Shannon Puloski, Kimberly Carcary.

Henderson Hospital, Hamilton, ON: Victoria Avram*. Kingston General Hospital, Kingston, ON: Ryan Bicknell*, Jeff Yach, Davide Bardana, Sue Lambert,

London Health Sciences Centre, London, ON: David W. Sanders*, Jamie Howard, Mark Macleod, C.T. Bartly, Christina Tieszer.

McMaster University Medical Centre, Hamilton, ON: Devin Peterson*. Oakville Trafalgar Memorial Hospital, Oakville, ON: Paul Zalzal*, Victor Maumetz, Heather Brien, Brad Weening.

Ottawa Hospital - Civic Campus, Ottawa, ON: Eugene K. Wai*, Darren Roffey. Royal Columbian Hospital, New Westminster, BC: Robert McCormack*, Trevor Stone, Bertrand Perey, Darius Viskontas, Dory Boyer, Bert Perey, Mauri Zomar, Karyn Moon, Amber Oatt.

St Michael's Hospital, Toronto, ON: Emil H. Schemitsch*, Michael McKee, Jeremy Hall, Henry Ahn, Milena R. Vicente, Lisa M. Wild.

Sunnybrook Health Sciences Centre, Toronto, ON: Hans J. Kreder*, David J.G. Stephen*, Markku Nousianinen, Ria Cagaanan, Monica Kunz.

Toronto Western Hospital, Toronto, ON: Khalid Syed*, Tania Azad. Queen Elizabeth II Health Sciences Centre, Halifax, NS: Chad Coles*, Ross Leighton*, David Johnstone, Mark Glazebrook, David Alexander, Kelly Trask, Gwendolyn Dobbin.

Investigators - The USA (site principal investigator is marked with an asterisk) Boone Hospital Center - Columbia Orthopaedic Group, Columbia, MO: Todd M Oliver*, Vicky Jones, James Ronan.

Boston University Medical Center, Boston, MA: Paul Tornetta II**, Desmond T. Brown, Hope Carlilse, Lisa Shaughnessy.

Colorado Orthopaedic Consultants, Aurora, CO: John Schwappach*, Craig A. Davis*, Peter Weingarten, Stewart Weinerman, Heike Newman, Janell Baker, Kieran Browner. Meghan Hurley.

Duke University Medical Center, Durham, NC: Robert Zura*, Maria J. Manson. Greenville Hospital System, Greenville, SC: Kyle Jeray*, David Goetz, Scott J. Broderick, Scott Porter, Thomas Pace, Stephanie L. Tanner, Becky Snider. Hennepin County Medical Center, Plymouth, MN: Andrew H. Schmidt* Jonathan Haas, David Templeman, Jerald R. Westberg. Indiana University - Wishard Health Services, Indianapolis, IN: Brian Mullis*, J. P. Ertl, Karl Shively, Valda Frizzel.

Lahey Clinic, Burlington, MA: Andrew J. Marcantonio*, Richard lorio*, Margaret Lobo, Michael Kain, Lawrence Specht, John Garfi. Miami Valley Hospital, Dayton, OH: Michael J. Prayson*, Craig Davis, Richard Laughlin, Joe Rubino, Mathew Lawless, Matt DiPaola, Chris Gaydon, Liz Dulaney.

MetroHealth Medical Center, Cleveland, OH: Heather A. Vallier*, John Wilber, John Sontich, Brendan Patterson, Andrea Dolenc, Chalitha Robinson, Roger Wilber. 
Mission Hospital Research Institute, Asheville, NC: Charles J. DePaolo*, Rachel Alosky, Leslie E Shell.

Northwest Orthopaedic Specialists, Spokane, WA: Jonathan P. Keeve*, Chris Anderson, Michael McDonald, Jodi Hoffman.

Ortholndy, Indianapolis, IN: Joseph Baele*, Tim Weber, Matt Edison, Dana Musapatika.

Orthopaedic Associates of Michigan, Grand Rapids, MI: Clifford Jones*, James Ringler, Terrance Endres, Martin Gelbke, Michael Jabara, Debra L. Sietsema, Susan M. Engerman.

Regions Hospital, St Paul, MN: Julie A. Switzer*, Mangnai Li, Scott Marston, Peter Cole, Sandy X. Vang, Amy Foley.

Santa Clara Valley Medical Center, San Jose, CA: Jessica McBeth*, Curt Comstock, Navid Ziran.

St Elizabeth Health Center, Youngstown, OH: James Shaer*, Barbara Hileman. St Louis University Hospital, St Louis, MO: David Karges*, Lisa Cannada*, Djoldas, Kuldjanov, John Tracy Watson, Emily Mills, Tiffany Simon.

Texas Tech University Health Sciences Center - El Paso, El Paso, TX: Amr Abdelgawad*, Juan Shunia.

Texas Tech University Health Sciences Center - Lubbock, Lubbock, TX: Mark Jenkins*, Mimi Zumwalt, Amanda West Romero.

University of Alabama, Birmingham, AL: Jason Lowe*, Jessica Goldstein.

University of California Irvine Medical Center, Orange, CA: David P.

Zamorano*, Deanna Lawson

University of Cincinnati Medical Center, Cincinnati, OH: Michael Archdeacon* John Wyrick, Shelley Hampton.

University of Connecticut, Hartford, CT: Courtland G. Lewis*, Arben Ademi, Raymond Sullivan, Stephanie Caminiti.

University of Mississippi Medical Center Jackson, MS: Matthew Graves*, Lori Smith.

University of Missouri Hospital and Clinics, Columbia, MO: Gregory J. Della Rocca*, Brett D. Crist, Yvonne Murtha, Linda K. Anderson, Toni K. Kliewer, Melinda K. McPherson, Kelly M. Sullivan.

University Orthopaedic Associates, New Brunswick, NJ: Carlos Sagebien*, Patricia Seuffert.

University of Pennsylvania, Philadelphia, PA: Samir Mehta*, John Esterhai, Jaimo Ahn, Fotios Tjoumakaris, Annamarie D. Horan, Christine Kaminski. University of Pittsburgh, Pittsburgh, PA: Ivan Tarkin*, Peter Siska, Arlene Luther, James Irrgang, Dana Farrell.

University of Rochester Medical Center, Rochester, NY: John T. Gorczyca* Jonathan M. Gross, Stephen Lloyd Kates, Jen Colosi, Nancy Hibsch, Krista Noble.

University of Texas Health Sciences Center, San Antonio, TX: Animesh Agarwal*, Rebecca Wright.

US Army Institute of Surgical Research and Brooke Army Medial Center, Fort Sam Houston, TX: Joseph R. Hsu*, James R. Ficke, Matthew A. Napierala, Michael T. Charlton, Mary K. Fan.

Vanderbilt University Medical Center, Nashville, TN: William T. Obremskey*, Justin E. Richards, Kenya Robinson.

Wake Forest Medical Center, Winston-Salem, NC: Eben Carroll*, Brenda Kulp. Funding

Members of the research team received a grant from Fonds NutsOhra (grant number T-0602-43), ZonMw (grant number 170.885.607), Physicians' Services Incorporated Foundation (grant number 08-18), Canadian Institutes of Health Research (grant number 177466) and National Institutes of Health (grant number 1R01-AR055267-01A1). The funding agencies were not involved in the study design, data collection, data analysis, manuscript preparations or publication decisions for this manuscript.

\section{Author details}

'Dept. of Surgery-Traumatology, Erasmus MC, University Medical Center Rotterdam, P.O. Box 2040, 3000 CA Rotterdam, the Netherlands. ${ }^{2}$ Dept. of Clinical Epidemiology and Biostatistics, McMaster University, HSC 2C, 1200 Main Street West, Hamilton, ON, L8N 3Z5, Canada. ${ }^{3}$ Dept. of Surgery, Kennemer Gasthuis, P.O. Box 417, 2000 AK, Haarlem, the Netherlands. ${ }^{4}$ Dept. of Orthopaedic Surgery, University of Minnesota Medical School, 2512 South $7^{\text {th }}$ Street, Suite R200, Minneapolis, MN, 55454, USA. ${ }^{5}$ Dept. of Emergency Medicine, Erasmus MC, University Medical Center Rotterdam, P.O. Box 2010, 3000 CA Rotterdam, the Netherlands.

\section{Authors' contributions}

The authors made the following contributions:
S.Z. (Dutch coordinator FAITH trial): study design, data acquisition and analysis, interpretation of data, drafting and critical revision of the manuscript, approved final version

H.V. (Team member methods center FAITH trial): data acquisition and interpretation of data, critical revision of the manuscript, approved final version

M.H. (Steering committee member FAITH trial): study design, interpretation of data, drafting and critical revision of manuscript, approved final version M.S. (Steering committee member FAITH trial): study design, interpretation of data, drafting and critical revision of manuscript, approved final version M.B. (Steering committee chair FAITH trial): study design, interpretation of data, drafting and critical revision of manuscript, approved final version P.P. (Site principal investigator FAITH trial): study design, interpretation of data, drafting and critical revision of manuscript, approved final version E.V.L. (Research coordinator Erasmus MC): study design, data analysis and interpretation of data, drafting and critical revision of the manuscript, approved final version

\section{Competing interests}

The authors declare that they have no competing interests.

Received: 17 October 2011 Accepted: 8 January 2012

Published: 8 January 2012

\section{References}

1. McLeod RS: Issues in surgical randomized controlled trials. World I Surg 1999, 23(12):1210-1214.

2. Trippel SB, Bosse MJ, Heck DA, Wright JG: Symposium. How to participate in orthopaedic randomized clinical trials. J Bone Joint Surg Am 2007, 89(8):1856-1864.

3. Thoma A, Farrokhyar F, McKnight L, Bhandari M: Practical tips for surgical research: how to optimize patient recruitment. Can J Surg 53(3):205-210.

4. Bossert EA, Evans S, Van Cleve L, Savedra MC: Multisite research: a systems approach. J Pediatr Nurs 2002, 17(1):38-48.

5. Bhandari $M$, Schemitsch EH: Beyond the basics: the organization and coordination of multicenter trials. Tech Orthop 2004, 19:83-87.

6. Farrell B, Kenyon S, Shakur H: Managing clinical trials. Trials 11:78.

7. Sprague S, Matta JM, Bhandari M, Dodgin D, Clark CR, Kregor P, Bradley G, Little L: Multicenter collaboration in observational research: improving generalizability and efficiency. J Bone Joint Surg Am 2009, 91 (Suppl 3):80-86.

8. Van Lieshout EMM, Zielinski SM: Surviving a site audit: tips for good clinical practice in an implant trial. J Long Term Eff Med Implants 2009, 19(3):215-222.

9. Kidd TJ, Marks GB, Bye PT, Wainwright CE, Robinson PJ, Rose BR, Harbour C, Bell SC: Multi-centre research in Australia: analysis of a recent National Health and Medical Research Council-funded project. Respirology 2009, 14(7):1051-1055.

10. van Santvoort HC, Besselink MG, Gooszen HG: [Obtaining medical ethical approval for a multicentre, randomised study: prospective evaluation of a ponderous process]. Ned Tijdschr Geneeskd 2008, 152(38):2077-2083.

11. Sherwood ML, Buchinsky FJ, Quigley MR, Donfack J, Choi SS, Conley SF, Derkay CS, Myer CM Jr, Ehrlich GD, Post JC: Unique challenges of obtaining regulatory approval for a multicenter protocol to study the genetics of RRP and suggested remedies. Otolaryngol Head Neck Surg 2006, 135(2):189-196.

12. Greene SM, Geiger AM: A review finds that multicenter studies face substantial challenges but strategies exist to achieve Institutional Review Board approval. J Clin Epidemiol 2006, 59(8):784-790.

13. Stair TO, Reed CR, Radeos MS, Koski G, Camargo CA: Variation in institutional review board responses to a standard protocol for a multicenter clinical trial. Acad Emerg Med 2001, 8(6):636-641.

14. Zurbuchen U, Schwenk W, Bussar-Maatz R, Wichlas F, Buhr HJ, Neudecker J, Ritz JP: [Clinical studies outside university clinics: What are the problems to implement this?]. Chirurg 81(2):160; 162-166.

15. Raftery J, Kerr C, Hawker S, Powell J: Paying clinicians to join clinical trials: a review of guidelines and interview study of trialists. Trials 2009, 10:15.

16. Jeray $\mathrm{K}$, Tanner $\mathrm{S}$, Schemitsch $\mathrm{EH}$, Bhandari M: Are large fracture trials possible? J Orthop Trauma 24(Suppl 1):S87-92.

17. Csimma C, Swiontkowski MF: Large clinical trials in musculoskeletal trauma: are they possible? Lessons learned from the international study 
of the use of rhBMP-2 in open tibial fractures. J Bone Joint Surg Am 2005, 87(1):218-222.

18. Irving SY, Curley MA: Challenges to conducting multicenter clinical research: ten points to consider. AACN Adv Crit Care 2008, 19(2):164-169.

19. Sprague $S$, Leece $P$, Bhandari M, Tornetta P Jr, Schemitsch E, Swiontkowski MF: Limiting loss to follow-up in a multicenter randomized trial in orthopedic surgery. Control Clin Trials 2003, 24(6):719-725.

20. Bhandari M: Clinical Research for Surgeons. Stuttgart: Thieme Medical Publishers: 12009

21. Bhandari M, Sprague S, Schemitsch EH: Resolving controversies in hip fracture care: the need for large collaborative trials in hip fractures. $J$ Orthop Trauma 2009, 23(6):479-484.

22. Bhandari M, Devereaux PJ, Tornetta P Jr, Swiontkowski MF, Berry DJ, Haidukewych G, Schemitsch EH, Hanson BP, Koval K, Dirschl D, et al: Operative management of displaced femoral neck fractures in elderly patients. An international survey. J Bone Joint Surg Am 2005, 87(9):2122-2130.

23. Abraham NS, Young JM, Solomon MJ: A systematic review of reasons for nonentry of eligible patients into surgical randomized controlled trials. Surgery 2006, 139(4):469-483.

24. Kooistra BW, Dijkman BG, Guyatt GH, Sprague S, Tornetta P Jr, Bhandari M: Prospectively screening for eligible patients was inaccurate in predicting patient recruitment of orthopedic randomized trials. J Clin Epidemiol .

doi:10.1186/1745-6215-13-5

Cite this article as: Zielinski et al:: Central coordination as an alternative for local coordination in a multicenter randomized controlled trial: the FAITH trial experience. Trials 2012 13:5.

\section{Submit your next manuscript to BioMed Central} and take full advantage of:

- Convenient online submission

- Thorough peer review

- No space constraints or color figure charges

- Immediate publication on acceptance

- Inclusion in PubMed, CAS, Scopus and Google Scholar

- Research which is freely available for redistribution

Submit your manuscript at www.biomedcentral.com/submit
Biomed Central 